\title{
Zwei neue Pseudoskorpion-Arten (Arachnida, Pseudoscorpiones) aus marokkanischen Höhlen
}

\author{
von \\ Volker MAHNERT*
}

\section{SUMMARY}

Two new Pseudoscorpion-species from moroccan caves

Chthonius (E.) longesetosus n. sp. (1) collected in the cave of Sidi Mejbur Tazas, and Allochernes maroccanus n. sp. (4 o 194 Tritonymphs), collected in the cave of Caïd (= Ifri el Caïd), Aït Mehammed, are described and figured. It is the first record of cave dwelling pseudoscorpions in Morocco aside from the publication of a Chthonius sp. from a cave near Taza.

Mein Kollege, Dr. B. Hauser (Département des Arthropodes) vertraute mir eine kleine Pseudoskorpion-Ausbeute zur Bearbeitung an, die Herr P. Strinati (Genf) auf einer kurzen Reise im Mai 1974 (gemeinsam mit Herrn Dr. J. M. Thibault, Brunoy) in marokkanischen Höhlen gesammelt und die er dem Genfer Museum überlassen hatte; beiden Herren danke ich herzlich. In dem Material befanden sich 2 Arten, beide erwiesen sich als neu für die Wissenschaft, nicht erstaunlich, da aus Marokko bisher kein einziger Höhlenpseudoskorpion beschrieben worden ist.

\section{Chthonius (E.) longesetosus n. sp. (Fig. 1-4)}

Fundort: Marokko, Grotte von Sidi Mejbeur, Taza, P. Strinati lg., 2.5.1974: 1 ( (Holotypus)

Beschreibung: Carapax (Fig. 1) etwas länger als in Augenniveau breit, kaudal mässig verengt, kein Epistom, Vorderrand in der Mitte etwas vorgezogen und gezähnelt, Vorderaugen mit ganz flacher Linse, Hinteraugen als undeutliche helle Flecken erkennbar, mit 22 langen Borsten und je 1 prä-

\footnotetext{
* Museum d'Histoire naturelle, case postale 284, CH-1211 Genève 6, Suisse.
} 

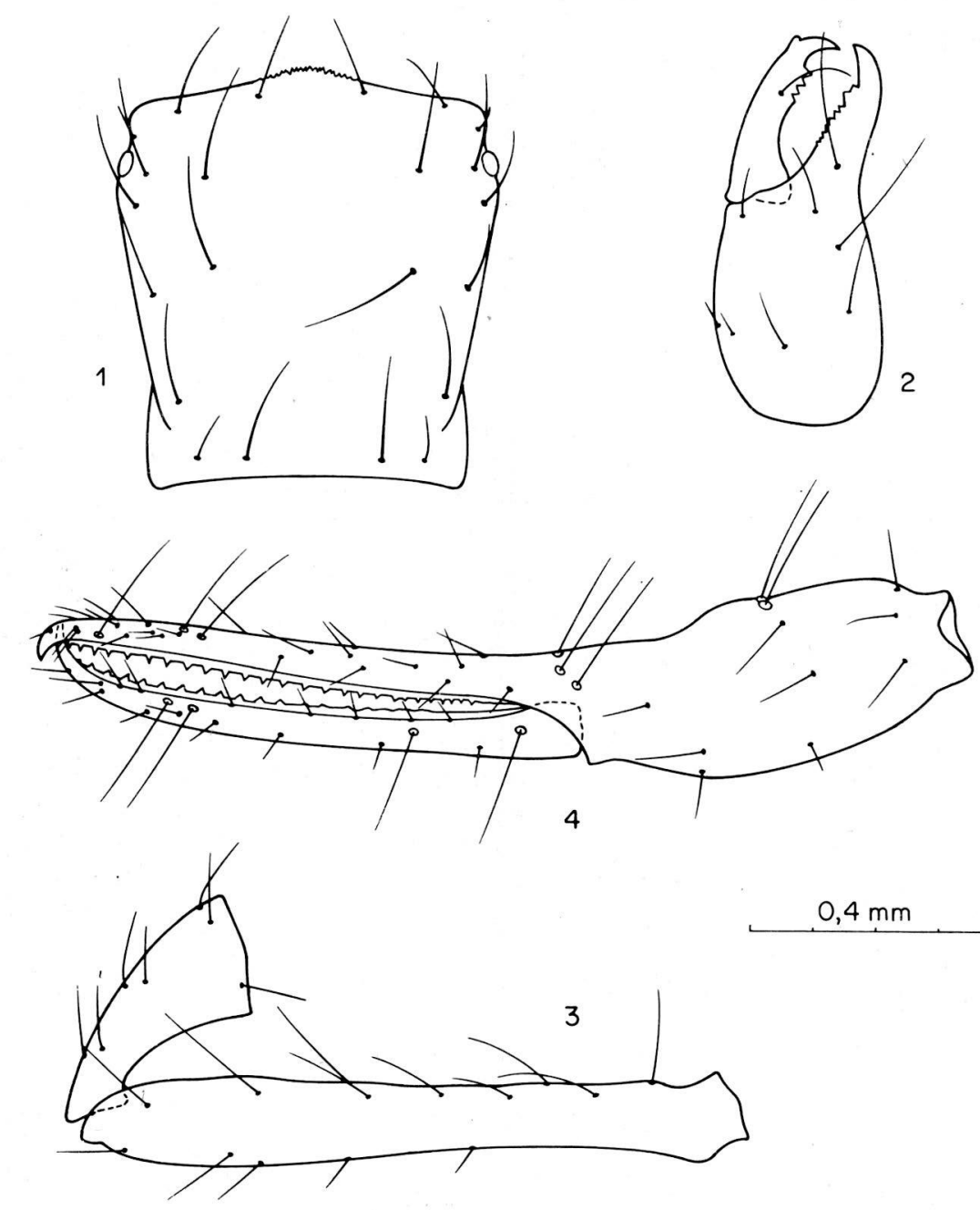

Fig. 1-4. Chthonius (E.) longesetosus n. sp.; 1: Carapax; 2: linke Chelicere; 3-4: Pedipalpe. 
okularen Mikrochaete, 2 lange und 2 kürzere Borsten am Hinterrand. Tergitbeborstung: 4-4-4-4-6-6-6-6-6-4-6, letztes Tergit mit 2 submedianen Tastborsten; Cheliceren (Fig. 2) mit 6 Stammborsten und 2 seitlichen Börstchen, fester Finger mit 2 derberen distalen Zähnen, anschliessend 7 kleinere, beweglicher Finger mit 4 proximalwärts kleiner werdenden Zähnen, Spinnhöcker klein, Serrula externa mit 17 Lamellen, Flagellum mit 11 einseitig gezähnten Borsten. Lobus der Pedipalpencoxen mit 2 Borsten, Pedipalpencoxa 3 Borsten, Coxa I $3+3$ marginale Börstchen, II $4+14$ Coxalborsten, III $5+$ ca. 10 Coxalborsten, IV 6, Intercoxaltuberkel mit 2 Borsten. Genitaloperkel mit 10 Borsten, Tergitbeborstung: $8+2 / 3$ Stigmenbörstchen -7+2 2 2-8-6-6-6-6-7; Sternite V-VII mit sehr kurzen Lateralborsten, Endsternit mit 2 submedianen Tastborsten. Pedipalpen (Fig. 3-4): Femur 7,42x länger als breit, Tibia 2,33x, Hand 2,30x, Schere 5,57x; Finger 1,42x länger als Hand, fester Finger mit 27 spitzen getrennt stehenden Zähnen, die basalen vier rudimentär, beweglicher Finger mit 12 spitzen, getrennt stehenden Zähnen, deren Reihe bis halbwegs zwischen st und $s b$ reicht, anschliessend noch 3 Rudimente (Zahnkanal sichtbar), dann leicht gewellte Zahnlamelle; Tasthaar ist auf gleicher Höhe mit esh stehend. Laufbein I: Femur I 7,29x länger als breit, Femur II 4,01x, Tibia 5,69x, Tarsus 12,0x, Femur I 2,08x länger als Femur II, Tarsus 1,77x länger als Tibia; Laufbein IV: Femur 2,82x, Tibia 5,31x, Tarsus I 3,58x, Tarsus II 13,90x länger als breit, Tarsus II mit Tastborste $(\mathrm{TS}=0,35)$, Tarsus II 2,05x länger als Tarsus I.

Körpermasse (in $\mathrm{mm}$ ): Körperlänge 2,07x; Carapax 0,63-0,58; Pedipalpen: Femur 1,02-0,14, Tibia 0,42-0,18, Hand 0,61-0,26, Schere 1,46-0,26, Finger-L. 0,86; Laufbein I: Femur I 0,57-0,08, Femur II 0,28-0,07, Tibia 0,33-0,06, Tarsus 0,58-0,05; Laufbein IV: Femur 0,81-0,29, Tibia 0,55-0,10, Tarsus I 0,28-0,08, Tarsus II 0,57-0,04.

Aus Nordafrika ist bislang nur eine Höhlenform, C. (E.) anophthalmus Ell. aus Algerien, bekannt geworden, die tetrachelatus (Preyssler) ähnlich sein soll und viel kleiner ist als longesetosus n. sp.; Strinati (1953) meldet Chthonius sp. aus der Gegend von Taza (Grotte von Ras el Oued), wobei es sich wahrscheinlich um die selbe Art handeln könnte. C. longesetosus n. sp. ist wahrscheinlich mit C. gibhus Beier am nächsten verwandt (Palpenhand distal von $i b / i s b$ mit leichter Beule), ist auf gleicher Höhe von esb, ähnliche Bezahnung der Palpenfinger), unterscheidet sich jedoch durch deutlich schlankere Palpenglieder, zahlreichere Zähne, bedeutendere Grösse und die Reduzierung der Augen.

\section{Allochernes maroccanus n. sp. (Fig. 5-11)}

Fundort: Marokko, Grotte du Caïd (= Ifri el Caïd), Aït Mehammed, P. Strinati lg., 4.5.1974: $1 \hat{\sigma}$ (Holotypus), $3 \hat{\delta} 1 q 4$ Tritonymphen (Paratypen). 
Beschreibung des ô: Carapax und Palpen rötlich braun, Tergite und Sternite gelblich braun, Pedipalpen, Carapax und Tergite grob granuliert, Vestituralborsten kurz, gezähnt und gekeult, Borsten der Beine gezähnt und leicht gekeult; Carapax etwas länger als am Hinterrand breit, grob granuliert, mit 2 deutlichen Querfurchen, wobei die subbasale dem Hinterrand deutlich näher liegt als der medianen; keine Augen oder Augenflecken, ca. 50-60 Borsten, wovon 4 am Vorder- und 8-10 am Hinterrand stehen; alle Tergite bis auf das letzte vollständig geteilt, Beborstung der Halbtergite: I meist mit 5 (einmal 7) Hinterrandborsten, die übrigen mit 4-9 (meist 6-7) Hinterrandborsten, die mittleren zusätzlich mit je einer lateralen (ab Tergit IV) und medialen (ab Tergit I) Randborste sowie einer medialen Diskalborste, Endtergit ohne Tastborsten; Cheliceren (Fig. 5) mit 5 Stammborsten, von denen $d b$ und $i b$ gezähnt sind (bei einem $\delta \hat{~} i b$ glatt), Galea mit 5-6 apikalen und subapikalen Aestchen, fester Finger mit 3 subapikalen Körnchen und 2-3 Zähnen, beweglicher Finger zahnlos, mit deutlichem Subapikallobus, Serrula externa mit 17-18 Lamellen, Flagellum aus 3 Borsten bestehend, die distale einseitig gezähnt (ca. 6-7 Zähnchen). Lobus der Pedipalpencoxen mit 3 Marginal- und 1 Diskalborsten,Pedipalpencoxen granuliert, mit ca. 20 Borsten, Coxa des Laufbeins I 11-13, II 13-18, III 13-21, IV 28-35; Genitaloperkel mit 32-41 Borsten, Genitalkammer mit 2 Gruppen von je 3-5 Börstchen, Tergite bis auf letztes vollständig geteilt, mit 6-10 Hinterrandborsten, Stigmata mit 2-3 Börstchen; Pedipalpen (Fig. 7-9): Femur aus dem Stielchen hinten schräg verdickt, Medialkante fast gerade, 3,98-4,18x länger als breit, Tibia sehr schlank, mit fast geraderLateralkontur, 3,24-3,34x, Hand mit Stiel 2,42-2,65x, Lateralkontur der Hand gerade, Schere mit Stiel 4,18-4,39x länger als breit, Finger deutlich kürzer als Hand ohne Stiel, fester Finger mit 46, beweglicher Finger mit 48-50 Zähnen, fester Finger medial mit 2 Nebenzähnen (1 weit distal, knapp proximal der Klaue), lateral mit deren 2-5, beweglicher Finger medial mit 1, lateral mit 1-3 Nebenzähnen; nodus ramosus im beweglichen Finger knapp distal von $s t$ liegend; Tasthaar st nur wenig näher bei $t$ als bei $s h$, eb-esh-ib-ish an der Basis gruppiert, ist stark an est genähert, it etwas näher bei et als bei ist. Laufbein I: Femur I, 1,79-1,87x länger als breit, Femur II 3,40-3,89x, Tibia 4,71-4,98x, Tarsus 6,88-7,32x, Femur II 1,54-1,71x länger als Femur I, Tarsus 1,06-1,12x länger als Tibia; Laufbein IV: Femur 4,33-4,87x länger als breit, Tibia 5,85-6,23x, Tarsus 6,74-6,89x, ohne Tastborste, Tibia 1,27-1,33x länger als Tarsus.

Beschreibung des $q$ : Chaetotaxie des Carapax, der Tergite und mittleren Sternite, der Coxen und der Cheliceren identisch mit der der Männchen; Coxa des Laufbeins IV mit ca. 50 Borsten, Genitaloperkel mit 32 Borsten (die Mitte freilassend), Sternit III mit 18 Hinterrandborsten und 3-5 Stigmenbörstchen; Serrula externa mit 17 Lamellen. Galea s. Fig. 6; Flagellum mit 3 Borsten, wovon die erste 7 , die zweite (!) 2 Zähnchen aufweist. Pedipalpen: Femur 3,97x länger als breit, Tibia 3,18x, Hand mit Stiel 2,50x, Schere mit Stiel 4,14x, Finger deutlich kürzer als Hand ohne Stiel; 


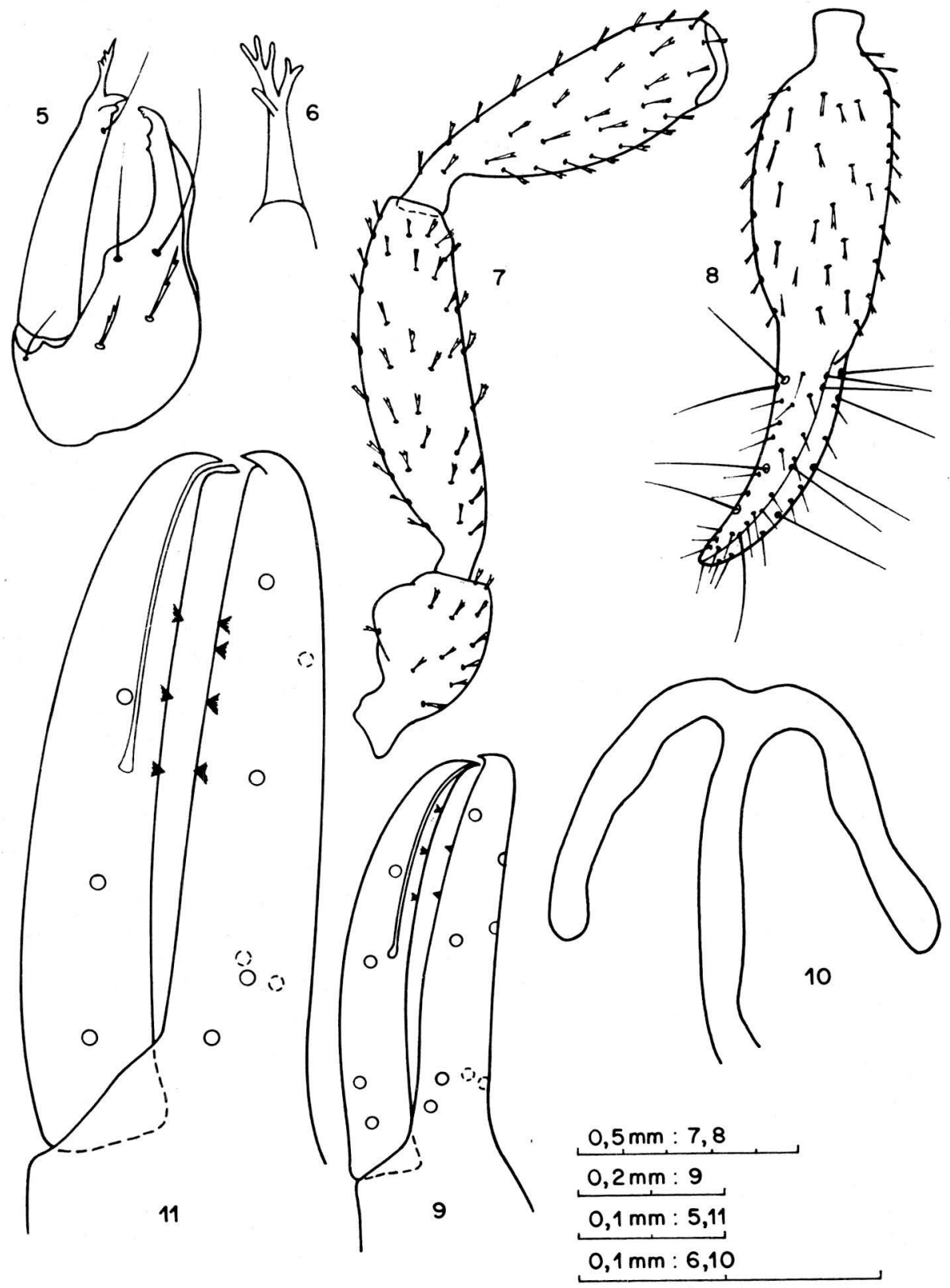

Fig. 5-11. Allochernes maroccanus n. sp.; 5: linke Chelicere (ठิ); 6: Galea (Q)); 7-9: Pedipalpe (ठ); 10: Spermathek ( + ); 11: Finger der linken Pedipalpenschere (Tritonymphe). 
fester Finger mit 45, beweglicher Finger mit 46 Zähnen, fester Finger lateral und medial mit je 2 Nebenzähnen, beweglicher Finger lateral mit 3, medial mit 1 Nebenzähnen. Laufbein I: Femur I 1,81x länger als breit, Femur II 3,44x Tibia 4,81x, Tarsus 6,78x, Femur II 1,53x länger als Femur I, Tibia (!) 1,01x länger als Tarsus; Laufbein IV: Femur 5,10x länger als breit, Tibia 6,09x, Tarsus 6,63x, Tibia 1,35x länger als Tarsus.

Körpermasse (in mm): Körperlänge 2,4-2,7 ( $\circ$ : 2,9); Carapạ 0,76-0,83/ 0,65-0,71 ( + 0,85/0,72); Pedipalpen: Femur 0,81-0,89/0,20-0,22 (0,91/0,23); Tibia $0,72-0,78 / 0,22-0,24(0,80 / 0,25) ; \quad H a n d m i t$ Stiel $0,67-0,73 / 0,28-0,30$ $(0,80 / 0,32)$, Stiel 0,09-0,11 (0,11); Finger-L. 0,53-0,59 (0,61), Scheren-L. mit Stiel 1,16-1,28 (1,33). Laufbein I: Femur I 0,22-0,23/0,12-0,13 $(0,27 / 0,15)$, Femur II 0,35-0,39/0,09-0, $11(0,41 / 0,12)$, Tibia 0,35-0,37/0,07-0,08 $(0,40 / 0,08)$, Tarsus 0,37-0,40/0,05-0,06 (0,40/0,08). Laufbein IV: Femur 0,65-0,69/0,14-0,16 $(0,78 / 0,15)$, Tibia $0,53-0,58 / 0,09-0,10(0,61 / 0,10)$, Tarsus $0,41-0,44 / 0,06$ $(0,46 / 0,07)$.

Beschreibung der Tritonymphe: Carapax wie bei Adulti; Halbtergite mit je 4-7 Hinterrandborsten, mittlere Tergite zusätzlich je eine mediale und laterale Rand- sowie eine Diskalborste; Cheliceren mit 4-5 Stammborsten (ib fehlt bei 1 Ex. auf der rechten Chelicere), $d h$ und ih gezähnt, Serrula externa mit 15-16 Lamellen, Flagellum mit 3 Borsten (distale gezähnt); Coxen der Pedipalpen mit 11-14 Borsten, Coxa des Laufbeins I 6-8, II 9-10, III 9-14, IV 14-24; Sternit II mit einer medialen Gruppe von 6-9 Borsten, Sternite mit 5-7 Borsten. Pedipalpen: Femur 3,40-3,56x länger als breit, Tibia 2,72-2,86x, Hand mit Stiel 2,30-2,43x, Schere mit Stiel 3,97-4,26x, Finger deutlich kürzer als Hand ohne Stiel; fester Finger mit 34-37, beweglicher Finger mit 37-41 Zähnen, fester Finger lateral mit 3-4, medial mit 2, beweglicher Finger lateral mit 1-3, medial mit 1 Nebenzähnen, nodus ramosus proximal von $t$ liegend (Fig. 11). Laufbein I: Femur 1,55-1,70x länger als breit, Femur II 2,91-3,02x, Tibia 3,69-3,84x, Tarsus 5,41-5,82x, Femur II 1,47-1,59x länger als Femur I, Tarsus 1,13-1,17x länger als Tibia; Laufbein IV: Femur 4,01-4,07x länger als breit, Tibia 4,63-4,89x, Tarsus 5,27-5,31x, Tibia 1,18-1,27x länger als Tarsus.

Körpermasse (in mm): Körperlänge 2,1-2,6; Carapax 0,58-0,72/0,52-0,64; Pedipalpen: Femur 0,52-0,65/0,15-0,19, Tibia 0,47-0,59/0,16-0,19, Hand mit Stiel 0,48-0,60/0,20-0,26, Stiel 0,07-0,09, Finger-L. 0,39-0,47, Scheren-L. 0,83-1,04; Laufbein I: Femur I 0,16-0,19/0,10-0,12, Femur II 0,23-0,30/ 0,05-0,06; Laufbein IV: 0,43-0,57/0,11-0,14, Tibia 0,35-0,44/0,07-0,09, Tarsus $0,29-0,36 / 0,06-0,07$.

Durch die sehr schlanken Palpenglieder an $A$. solarii (Simon) und tripolitanus Beier erinnernd, von diesen beiden Arten jedoch durch die deutlich kürzeren Palpenfinger und die Stellung des Tasthaars ist distal von est unterscheidbar. 
Von tripolitanus unterscheidet sich die neue Art darüberhinaus noch durch die Form des Palpenfemurs und durch bedeutendere Körpergrösse, von solarii durch deutlich schlankeres Palpenfemur und -tibia sowie durch die geringere Zahl von Nebenzähnen. Von powelli (Kew), mit dem maroccanus n. sp. in der Form der Palpen und in der Fingerlänge (kürzer als die Hand ohne Stiel) übereinstimmt, unterscheidet sich die neue Art sogleich durch die schlankeren Palpen und die Körpergrösse.

\section{ZUSAMMENFASSUNG}

Chthonius (E.) longesetosus n. sp. (1 $q$, Höhle von Sidi Mejbeur, Taza) und Allochernes maroccanus n. sp. (4 ô 1 \& 4 Tritonymphen; Höhle des Caïd (= Ifni el Caïd), Aït Mehammed) werden beschrieben und abgebildet. Beide Arten nehmen eine relativ isolierte Stellung ein, C. longesetosus könnte eine von $C$. gibbus abzuleitende Art darstellen, A. maroccanus ähnelt durch die schlanken Palpen den Arten solarii (Simon) und tripolitanus Beier, ist aber wahrscheinlich mit powelli (Kew) am nächsten verwandt. Abgesehen von der Meldung eines Chthonius sp. aus der Höhle von Ras el Oued, Taza, ist bislang keine höhlenbewohnende Pseudoskorpion-Art aus Marokko gemeldet.

\section{LITERATUR}

BEIER, M. 1954. Einige neue Pseudoscorpione aus dem Genueser Museum. - Annali Mus. civ. Stor. nat. Giacomo Doria, 66: 324-330.

BEIER, M. 1963. Ordnung Pseudoscorpionidea (Afterskorpione). - Bestimm. Bücher Bodenfauna Europas 1: $313 \mathrm{~S}$.

ELlingSEN, E. 1903. Biospéologica. VII. Pseudoscorpiones (seconde série). - Archs. Zool. exp. gén., sér. 4, 8: 415-420.

STRINATI, P. 1953. Campagne d’explorations spéléologiques au Maroc (été 1950). - Annls. Spéléol. 7: 99-107 (1952).

STRINATI, P. et V. AELLEN. 1959. Faune cavernicole de la région de Taza (Maroc.). Revue suisse Zool. 66: 765-777. 